ज्ञ FRANÇAISE

$\supset$ DE

띨 PÉDAGOGIE

\section{Revue française de pédagogie}

Recherches en éducation

157 | octobre-décembre 2006

PISA : analyses secondaires, questions et débats

théoriques et méthodologiques

\title{
Définition des compétences, choix méthodologiques et retombées sur la politique scolaire en Suisse
}

Definition of competencies, methodological choices and repercussions on school policy in Switzerland

Definición de las competencias, elecciones metodológicas y repercusiones sobre la política escolar en Suiza

Definition des Konzeptbegriffs, methodologisches Vorgehen und Auswirkungen

auf die Schweizer Schulpolitik

Jean Moreau, Christian Nidegger et Anne Soussi

\section{OpenEdition}

Journals

Édition électronique

URL : http://journals.openedition.org/rfp/415

DOI : $10.4000 /$ rfp. 415

ISSN : 2105-2913

Éditeur

ENS Éditions

Édition imprimée

Date de publication : 1 décembre 2006

Pagination : 43-53

ISBN : 978-2-7342-1075-7

ISSN : 0556-7807

\section{Référence électronique}

Jean Moreau, Christian Nidegger et Anne Soussi, « Définition des compétences, choix

méthodologiques et retombées sur la politique scolaire en Suisse », Revue française de pédagogie [En

ligne], 157 | octobre-décembre 2006, mis en ligne le 01 décembre 2010, consulté le 30 avril 2019.

URL : http://journals.openedition.org/rfp/415; DOI : 10.4000/rfp.415 


\section{Définition des compétences, choix méthodologiques et retombées sur la politique scolaire en Suisse}

Jean Moreau, Christian Nidegger, Anne Soussi

Nous essayerons dans le présent article de mettre en évidence, en illustrant par quelques exemples, les choix méthodologiques et conceptuels pris en compte dans l'enquête PISA. Dans quelle mesure les compétences définies et opérationnalisées permettent-elles la comparaison des systèmes éducatifs ou n'introduisent-elles pas de biais culturels et linguistiques ? Les méthodes statistiques sophistiquées utilisées permettent-elles la transparence et l'appropriation des données par les destinataires des résultats : décideurs politiques et acteurs de l'école? Dans une deuxième partie, nous montrerons les effets des résultats de PISA sur la politique scolaire en Suisse et plus particulièrement sur la mise en place d'un projet d'harmonisation scolaire national (HarmoS) qui s'inspire largement de démarches mises en œuvre dans l'enquête PISA, notamment approche par compétences, malgré des objectifs différents tels que le pilotage et la régulation des systèmes éducatifs.

Descripteurs (TEE) : compétence, évaluation, mesure, politique de l'éducation, Suisse.

'enquête PISA vise à mesurer, tous les trois ans, les compétences dans trois domaines, littéracie, culture mathématique et culture scientifique. En 2003, cette enquête a concerné plus de quarante pays dont la Suisse. Avec des résultats relativement moyens en littéracie et des résultats au-dessus de la moyenne en mathématiques, ce pays qui se caractérise par la coexistence de trois régions linguistiques et de vingt-six systèmes scolaires différents, a subi un électrochoc (comme d'autres pays, par ailleurs) En effet, la Suisse qui avait jusqu'alors peu participé aux enquêtes internationales était persuadée de l'excellence de son système scolaire. Des mesures ont été mises en place au plan national suite aux résultats de l'enquête PISA 2000.

Nous allons dans le présent article essayer de mettre en évidence, en illustrant par quelques exemples, les choix méthodologiques et conceptuels pris en compte dans l'enquête PISA. Ainsi, un choix a été fait par les concepteurs, de mesurer des compétences qui seront utiles «tout au long de la vie ». Ce choix pose un certain nombre de questions: dans quelle mesure les compétences définies et opérationnalisées permettent-elles la comparaison de systèmes éducatifs différents et n'introduisent-elles pas 
de biais culturels et linguistiques? Les méthodes statistiques sophistiquées utilisées permettent-elles la transparence et l'appropriation des données par les destinataires des résultats : décideurs politiques, acteurs de l'école (directions des écoles, enseignants, parents) ?

Dans une deuxième partie, nous montrerons les effets des résultats de PISA sur la politique scolaire en Suisse et plus particulièrement sur la mise en place d'un projet d'harmonisation scolaire national (« HarmoS») se caractérisant notamment par l'entrée à l'école à quatre ans et l'élaboration de standards minimaux de compétences dans quatre domaines scolaires, Langue I (langue d'enseignement), Langue II (première langue étrangère), mathématiques et sciences, dont trois sont identiques à ceux de PISA, et ceci à trois moments-clés du cursus scolaire, $2^{\mathrm{e}}$, $6^{\mathrm{e}}$ et $9^{\mathrm{e}}$ années. II est à noter que ce projet s'inspire largement de démarches utilisées lors de la mise en œuvre de l'enquête PISA, approche par compétences et méthodologie statistique de même nature.

\section{PISA : PROBLÈMES LIÉS À UNE UTILISATION LOCALE}

\section{Construction du matériel de test}

Pour constituer le matériel nécessaire à l'enquête, et assurer une diversité linguistique et culturelle, des directives précises ont été définies afin que chaque pays puisse proposer des items. En fait, pour l'enquête principale 2003 dans le domaine des mathématiques, plus de $60 \%$ des items proviennent de trois instituts (australien américain et néerlandais) chargés de l'enquête, quelques items issus de l'enquête TIMSS. Les $40 \%$ restant proviennent d'une douzaine de pays. L'ensemble des items proposés par ces pays a été adapté par ces instituts. Un groupe d'experts internationaux a assuré la supervision de l'ensemble. On notera que plus de $70 \%$ des items ont été proposés en anglais, $14 \%$ en néerlandais (provenant d'un des instituts pilotant l'enquête). Les autres items ont été proposés en allemand, espagnol, japonais, tchèque et français. L'ensemble du matériel retenu a été soumis aux pays participants afin qu'ils puissent faire part de leurs remarques et de leurs éventuels désaccords.

Un point important à respecter pour la mise en œuvre d'une enquête internationale à visée comparative est la question de la traduction et de l'adaptation du matériel. Pour ce faire, PISA a conçu un système de traduction à partir de deux sources (l'une en anglais et l'autre en français). Les pays étaient ensuite invités à harmoniser les deux traductions. Cependant, la traduction à partir de deux sources n'a pas toujours pu être respectée. De plus, chaque pays pouvait proposer des adaptations nationales afin de s'assurer que les textes proposés soient plus proches du contexte linguistique local. Toutefois, c'est bien le même matériel qui est soumis aux élèves. Ainsi, dans le but de la comparaison, la diversité culturelle est bien envisagée comme une difficulté à surmonter et à réduire et non comme un élément constitutif du domaine à mesurer. Cela peut avoir pour conséquence que pour garantir les possibilités de comparaison, on définisse un plus petit dénominateur commun qui soit éloigné de toutes les situations locales existantes.

Lors de chaque prise d'information, un des trois domaines est particulièrement étudié. À cet effet, un certain nombre d'échelles sont construites afin de mieux décrire les compétences des élèves dans ce domaine. Pour l'enquête 2003, dont le domaine principal était les mathématiques, il avait été prévu au départ de constituer des échelles de type « compétences " manifestées par les élèves (reproduction, connexions, réflexion), comparables aux sous échelles utilisées pour la littéracie en 2000 (retrouver l'information, développer une interprétation et réfléchir sur le texte). Cependant, il s'est avéré que ces échelles étaient non seulement trop fortement corrélées mais aussi hiérarchiques. C'est pourquoi les concepteurs ont opté pour des échelles qui reflétaient différents aspects des contenus mathématiques. Dans le tableau (page suivante), on notera que si ces différentes échelles comprennent à peu près le même nombre d'items, le type de questions varie toutefois, considérablement d'une échelle à l'autre. Les résultats des élèves pourraient alors dépendre plus du type de question que du contenu de l'échelle. Or, on sait d'expérience qu'il est potentiellement plus facile de répondre à une question à choix multiple que de formuler soi-même une réponse. Dans l'enquête PISA 2000 sur la littéracie, nous avions pu vérifier cet effet (Broi et al., 2003).

\section{Adéquation du modèle statistique aux analyses au niveau local}

La validation du matériel a été réalisée à partir d'un double processus : 1) empirique par des experts qui ont classé l'ensemble du matériel selon les objectifs visés et la difficulté attendue des items ; 2) statistique sur la 
Tableau 1. - PISA 2003 : items de mathématiques (répartition selon le type de contenu et le format des items)

\begin{tabular}{|l|c|c|c|c|}
\hline \multirow{2}{*}{ Type de contenu } & \multicolumn{4}{|c|}{ Format des items } \\
\cline { 2 - 5 } & $\begin{array}{c}\text { Items } \\
\text { à choix multiple }\end{array}$ & $\begin{array}{c}\text { Items } \\
\text { à réponse construite } \\
\text { fermée }\end{array}$ & $\begin{array}{c}\text { Items } \\
\text { à réponse construite } \\
\text { ouverte }\end{array}$ & Total \\
\hline Espace et formes & 8 & 6 & 6 & 20 \\
\hline Quantité & 6 & 2 & 15 & 23 \\
\hline $\begin{array}{l}\text { Variations } \\
\text { et relations }\end{array}$ & 3 & 4 & 15 & 22 \\
\hline Incertitude & 11 & 1 & 8 & 20 \\
\hline Total & 28 & 13 & 44 & 85 \\
\hline
\end{tabular}

Source : OCDE, PISA 2003, Technical Report, p. 29 (traduit et adapté par nos soins).

base des résultats au pré-test réalisé dans l'ensemble des pays participants à l'enquête, l'année précédant le recueil de données principal. Une série d'aller-retour entre ces processus a permis de sélectionner le matériel qui sera retenu pour l'enquête principale.

La mesure des compétences des élèves implique la prise en compte de nombreux aspects. Ces aspects concernent bien sûr l'instrument lui même : le choix des textes et des différents items proposés aux élèves, mais aussi la manière dont les réponses aux questions vont être utilisées pour produire des scores. Le choix d'un modèle par les concepteurs du projet (le modèle de Rasch) a des conséquences sur les évaluations des compétences moyennes des pays participant à cette enquête. Rappelons que ce modèle (cas particulier des modèles de réponse à l'item) définit la relation entre la compétence de l'élève, la difficulté de l'item et sa probabilité de réussir correctement cet item. Cette relation est exprimée par une fonction mathématique (la fonction caractéristique de l'item) et elle est représentée par une courbe en forme de $S$. Tous les items sont supposés avoir le même pouvoir discriminatif. Les compétences des élèves et les difficultés des items alors sont situées sur le même continuum (1).

On peut donc s'interroger sur certaines conséquences liées au choix du modèle. Tout d'abord, la difficulté d'une question telle qu'elle est définie par le modèle sur le plan international est-elle cohérente avec les différentes réalités locales ? En particulier des questions de difficulté équivalente ont-elles des réussites identiques au niveau local, la Suisse romande dans notre exemple? En outre, la difficulté d'un item, telle qu'elle transparaît sur le plan statistique, est-elle compatible avec sa définition critérielle par rapport au contenu de chaque tâche ?

Rappelons en effet, que différentes analyses statistiques de l'épreuve ont été mises en œuvre pour s'assurer des propriétés psychométriques des items dans chaque pays, en particulier : leur discrimination, leur difficulté et leur adéquation avec le modèle. Ces propriétés ne doivent pas trop différer entre les pays. Un item peut en effet être supprimé si ses caractéristiques sont insuffisantes.

Malgré ces précautions méthodologiques, on remarque que la calibration des items n'assure pas l'homogénéité des niveaux de difficulté des items dans un même pays. $\mathrm{Si}$, par exemple, on compare le pourcentage de réussite de chaque item pour deux cantons suisses, Fribourg et Genève (items de lecture pour PISA 2000), avec sa difficulté telle qu'elle est évaluée par le modèle, on constate (voir figure page suivante) que certains items de niveau, de difficulté identique selon le modèle statistique, obtiennent néanmoins des pourcentages de réussite très différents dans une même population scolaire. Le calibrage du modèle au niveau international n'a donc pas nécessairement une pertinence pour une évaluation locale des compétences. Cette distorsion introduite par le calibrage international entre les évaluations des difficultés des items peut différer d'une population à l'autre. La comparaison des deux nuages de points montre qu'il ne s'agit pas d'une simple translation des nuages. 


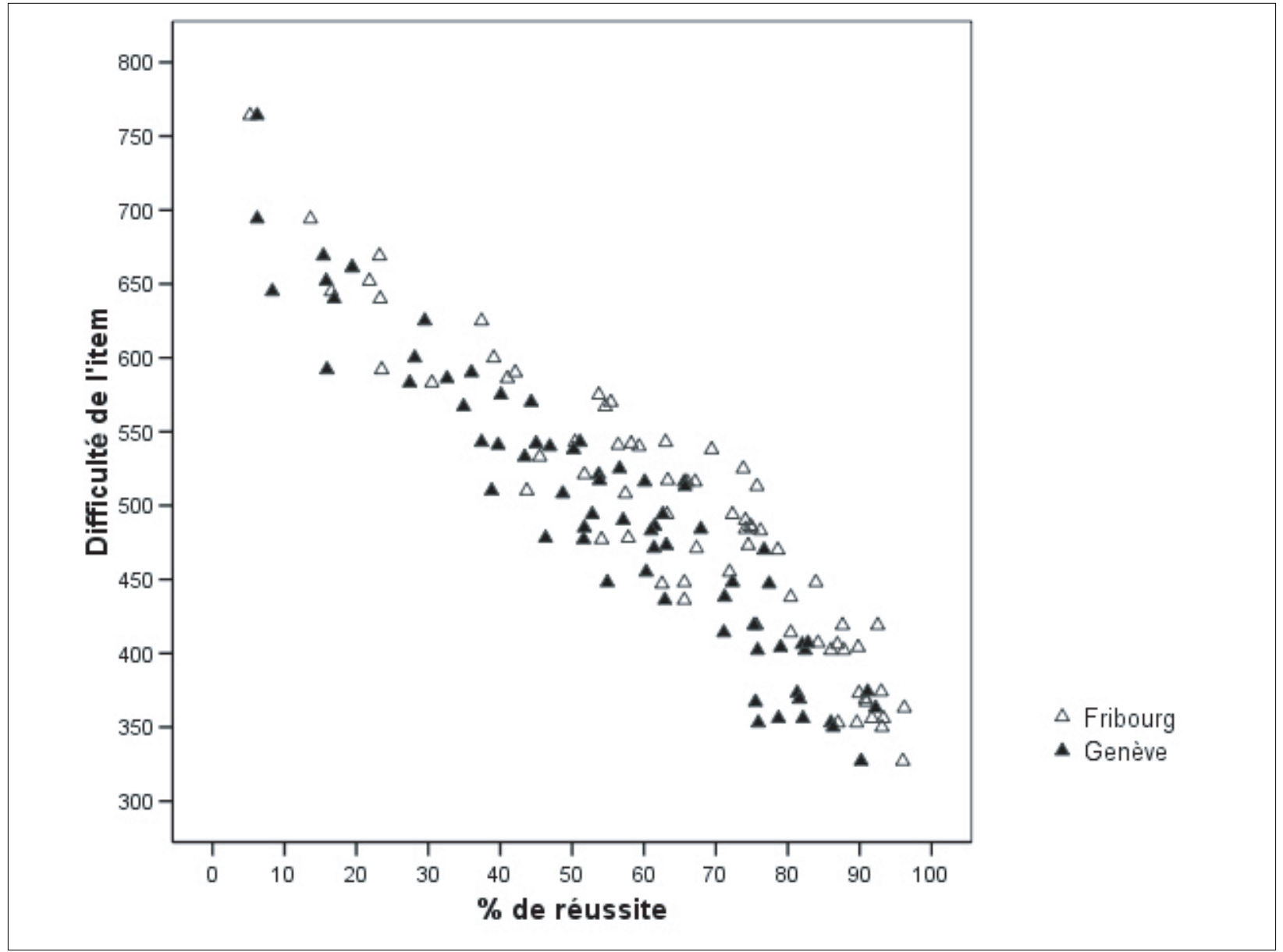

NB : pour des raisons de lisibilité, seuls $50 \%$ des items (choisis aléatoirement) sont représentés dans le graphique ci-dessus.

\section{Utilisation du modèle de Rasch pour l'évaluation des élèves}

L'enquête PISA n'a pas pour objectif l'évaluation des performances de chaque élève, mais plutôt la comparaison de systèmes éducatifs. Pourtant la tentation est grande d'utiliser sans précaution la même méthodologie pour évaluer les élèves. En effet, le modèle choisi permet à la fois d'estimer la difficulté des items et de produire des scores pour chaque élève.

Les différentes tâches proposées aux élèves sont en effet hiérarchisées en fonction de critères de difficulté croissante et devraient permettre de posi- tionner les élèves sur une échelle de Guttman. Dans une échelle de Guttman, un élève qui répond correctement à une question doit également donner une réponse correcte à toutes les questions plus faciles. On constate cependant qu'un élève qui réussit un item de difficulté donné peut échouer à des items plus faciles. En outre, un nombre important d'élèves de niveau faible (par exemple, niveau 1) réussissent des items de difficultés élevées (niveau 4 ou 5). En fait, conséquence directe du modèle de Rasch, aucun élève, même parmi les moins performants, n'a une probabilité nulle de répondre correctement à un item, même un item difficile. Des élèves peu performants ont donc pu réussir des items de niveau élevé. On constate, par exemple (PISA 2000) qu'un nombre 
important d'entre eux (plus de $50 \%$ des élèves de niveau 1) répondent correctement à au moins une question difficile (niveau 4 ou 5). On comprend que les élèves les moins performants sont des élèves qui n'ont répondu correctement qu'à peu de questions et surtout à des questions faciles.

On doit également remarquer que pour un tel modèle, des élèves avec des profils de réponses différentes ont pu obtenir le même score. En effet, le score de l'élève ne dépend pour un cahier donné que du nombre d'items réussis (score brut) et non de la difficulté des items réussis. Deux élèves qui répondent correctement au même nombre de questions - du même cahier (2) - obtiennent le même score quelle que soit la difficulté des questions auxquelles ils ont répondu. Autre conséquence surprenante, un élève peut être classé dans un niveau de lecture donné sans être confronté à des items de ce niveau. Par exemple, des élèves ont pu obtenir un niveau 5 sans être confrontés à des items de niveau 5 . Tout dépend du nombre d'items réussis. Certains cahiers, en effet, ne présentent aucun item de niveau 5 concernant l'interprétation du texte. Pourtant des élèves confrontés à ces cahiers ont obtenu le niveau 5 .

\section{Utilisation des indices internationaux au niveau régional : l'exemple du niveau socioéconomique}

Les performances des élèves sont mises en relation avec un certain nombre d'indices comme par exemple un indice de niveau socioéconomique. De notre point de vue, cet indice pose des questions au niveau de son utilisation pour des analyses régionales. En effet, dans la plupart des études, on observe une relation entre l'indice socioéconomique et la performance des élèves. Ce phénomène est également mis en évidence dans PISA 2003 (OCDE, 2004, chapitre 4). Par contre, lorsqu'au niveau régional on met en regard le niveau socioéconomique moyen et la performance des élèves, on s'aperçoit que ce ne sont pas les cantons qui ont le niveau socioéconomique moyen le plus élevé qui obtiennent la performance moyenne la meilleure (voir graphique 2).

Comment expliquer ce phénomène ? On peut par exemple se demander si la moyenne occulte des structures différentes des niveaux socioéconomiques entre les cantons. Le graphique 3 montre que si l'on répartit les élèves en quatre groupes en fonction de leur niveau socioéconomique, on observe par exemple que Genève a plus d'élèves de niveaux 3 et 4 que des deux autres niveaux (1 et 2) comparativement aux autres cantons. Ceci nous amène à faire l'hypothèse

\section{Graphique 2. - Moyenne en mathématiques et niveau socioéconomique (NSE) moyen}

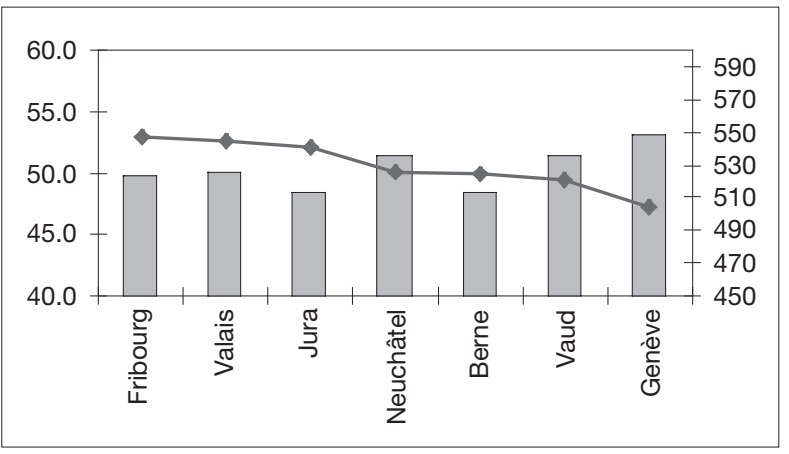

N.B. : Les histogrammes représentent le niveau socioéconomique (NSE) moyen (échelle de gauche), La courbe indique les scores moyens en mathématiques (échelle de droite).

d'une distorsion possible de cet indice international qui «favoriserait " les professions des services en leur attribuant une valeur d'indice plus élevé qu'à des professions «artisanales » qui exigent des qualifications égales ou supérieures et qui sont plus représentées dans les cantons moins urbains. Ainsi, le niveau socioéconomique moyen des cantons urbains serait en quelque sorte surévalué par une forte présence de professions donnant une valeur élevée d'indice bien que ne nécessitant pas de grandes qualifications. À l'inverse, le niveau socioéconomique moyen des cantons moins urbains (Fribourg, Valais, Jura et Berne) aurait tendance à être sous-évalué compte tenu de la proportion plus grande de professions donnant lieu à une valeur de cet indice plus faible malgré des professions à qualifications relativement élevées.

Par contre lorsque l'on met en relation la moyenne du niveau socioéconomique et les performances à l'intérieur des cantons en fonction de l'orientation des élèves, on constate bien une hiérarchie des performances en fonction de cet indice du niveau socioéconomique. En effet, la relation entre performance et niveau socioéconomique semble bien réelle mais l'indice utilisé par PISA n'est pas pertinent pour certaines comparaisons entre cantons. Ainsi lorsque l'on travaille sur les données à un niveau local, il est indispensable de se poser la question de la pertinence ou de la distance entre les instruments, conçus pour une comparaison à large échelle, et leurs exploitations locales afin d'éviter des interprétations abusives ou incohérentes des résultats observés. 


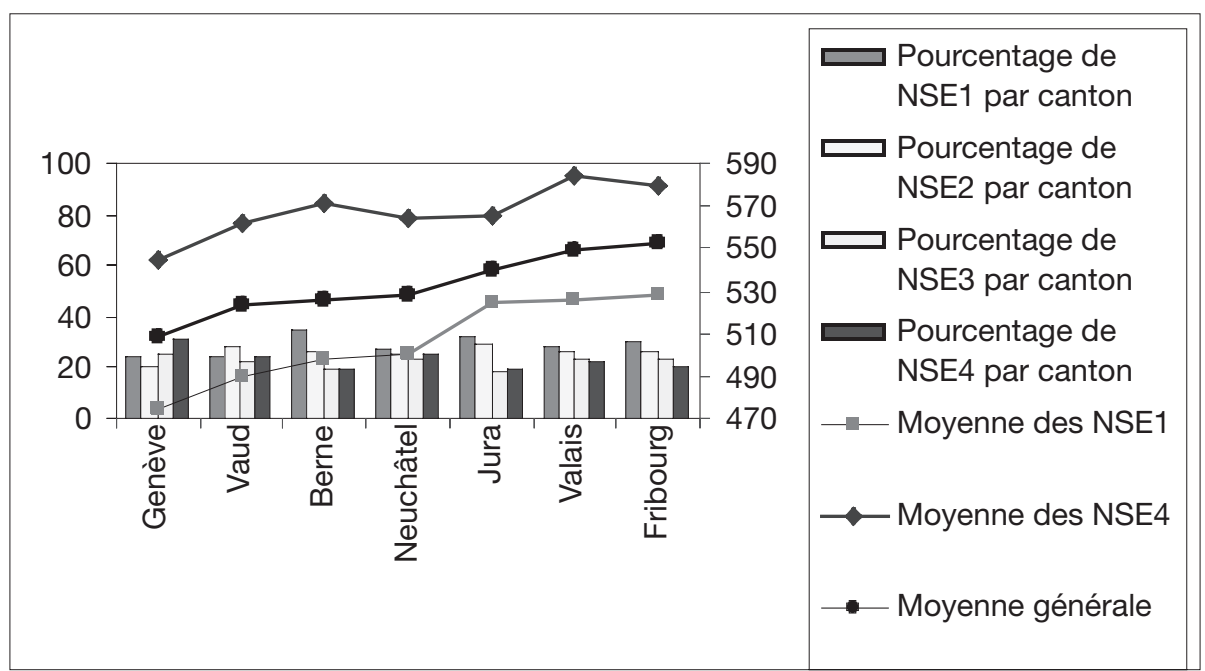

N.B. : Pour le niveau socioéconomique (NSE), les élèves de la Suisse romande ont été répartis en quatre catégories égales (de la plus basse à la plus élevée).

\section{RÉPERCUSSIONS SUR LA POLITIQUE EN SUISSE ET MODĖLES DE COMPÉTENCES}

Suite aux premiers résultats assez moyens obtenus à l'enquête PISA 2000, la Suisse par l'intermédiaire de la Conférence des directeurs de l'instruction publique (3) a décidé de mettre en place un certain nombre de mesures dont le lien avec PISA n'est pas toujours évident. Au plan cantonal, de nombreux plans d'action visant à développer et renforcer les compétences en lecture voire en littéracie ont été élaborés.

La Suisse est un système fédéral avec vingt-six cantons et autant de systèmes éducatifs et comporte trois régions linguistiques (germanophone, francophone, italophone). Ainsi, selon les cantons, qui sont responsables et souverains en matière scolaire, l'âge d'entrée à l'école n'est pas le même, le découpage entre les différents niveaux d'enseignement (primaire, secondaire I, etc.) est variable ainsi que la dotation horaire pour les différentes disciplines d'enseignement. Dans la liste des mesures proposées au niveau fédéral, on trouve un ambitieux projet d'harmonisation scolaire national (HarmoS) dont les principales propositions sont les suivantes:

- entrée dans l'école obligatoire à quatre ans révolus ;

- unification des finalités de l'école obligatoire ;
- description des compétences de base que doivent atteindre tous les élèves à la fin de la $2^{\mathrm{e}}, 6^{\mathrm{e}}$ et $9^{\mathrm{e}}$ années scolaires, au moyen de standards nationaux de formation ;

- élaboration d'un plan d'étude cadre pour chaque région linguistique ;

- vérification régulière au niveau du système scolaire de l'atteinte des standards (et mesures de remédiation mises en œuvre si nécessaire).

De manière générale, le projet HarmoS a pour objectifs d'harmoniser les contenus de l'enseignement, de renforcer le pilotage coordonné de la scolarité obligatoire, d'évaluer le système de formation à l'échelle nationale et de développer la qualité de l'enseignement. Outre la définition de standards que nous développerons plus en détail, Harmos prévoit d'autres instruments de pilotage tels que le monitorage, les plans d'étude et moyens d'enseignement ainsi que la formation des enseignants. Le monitorage vise à recueillir des données systématiques sur le système éducatif et son environnement, notamment au moyen de tests standardisés effectués régulièrement pour fournir des informations plus fiables sur la réussite, la qualité et la faiblesse du système et constitue un instrument de travail pour les responsables politiques permettant une prise de décision pour son pilotage. Concernant plans d'étude, moyens d'enseignement 
et formation des enseignants, on cherche à mettre en place une coordination, notamment par région linguistique, et une professionnalisation dans le cas de la formation des enseignants afin d'améliorer la qualité de l'enseignement.

\section{Modèles de compétences et élaboration de standards}

Dans cette première phase de l'harmonisation, la définition de standards et l'élaboration de modèles de compétences concernent seulement quatre domaines de compétences: la langue I (ou d'enseignement), les langues II, les mathématiques et les sciences. S'il s'agit de domaines pour lesquels la dotation horaire est relativement importante tout au long de la scolarité, on peut aussi observer une certaine ressemblance avec les domaines mesurés dans PISA (littéracie, culture mathématique et culture scientifique). Comme on peut le constater, ce projet au travers de modèles de compétences s'inscrit dans les courants actuels en éducation. Comme le relève $M$. Crahay (2006), on est passé de pédagogie par objectifs à une logique de la compétence.

Les responsables au niveau national ont réuni des experts de différents pays dont $\mathrm{E}$. Klieme qui a fait un certain nombre de propositions en vue d'élaborer des standards sur la base de l'expérience menée en Allemagne (initiée elle aussi après les résultats de l'enquête PISA 2000).

Le projet suisse repose sur des postulats définis par E. Klieme et al. (2003, p. 22). La compétence est définie de la manière suivante : « En accord avec Weinert (2001, p. 27), nous entendons par compétences les capacités et aptitudes cognitives dont l'individu dispose ou qu'il peut acquérir pour résoudre des problèmes précis, ainsi que les dispositions motivationnelles, volitives et sociales qui s'y rattachent pour utiliser avec succès et responsabilité les résolutions de problèmes dans des situations variables. "

Les compétences sont donc considérées ici comme des dispositions, "c'est-à-dire comme des programmes des représentations, des mécanismes et des capacités internes qui déterminent les comportements et réalisations observables des individus » (Klieme et al., 2003, p. 152).

Le concept de compétence a de nombreuses orientations, notamment dans le monde francophone. D'autres définitions existent dans le monde francophone. Ainsi, L. Allal (1999), la définit de la manière suivante: c'est « un réseau intégré et fonctionnel formé de composantes cognitives, affectives, sociales, sensorimotrices, susceptible d'être mobilisé en actions finalisées face à une famille de situations et fondé sur l'appropriation de modes d'interaction et d'outils socioculturels. " D'autres auteurs comme $X$. Roegier et P. Joannaert en ont une représentation assez proche. Le second ajoute toutefois un élément intéressant: «Une compétence suppose, au-delà du traitement efficace, que ce même sujet pose un regard critique sur les résultats de ce traitement qui doit être socialement acceptable. » (2002, p. 41). II introduit l'idée d'un regard critique du sujet qui doit non seulement mobiliser différentes ressources mais aussi les sélectionner et les coordonner.

Pour B. Rey et al. (2003), face à ce magma d'acceptions du terme compétences, il est nécessaire d'introduire des niveaux. Certaines sont dites de premier degré (procédures de base), d'autres de deuxième degré où il s'agit de posséder des procédures de base et de savoir sélectionner la bonne dans une situation donnée. Le troisième type, compétence de troisième degré, consiste à combiner plusieurs procédures de base face à une situation nouvelle et complexe.

Enfin, une autre représentation du concept vient du monde du travail. Pour G. Le Boterf (1995), une compétence est " un savoir-agir, c'est-à-dire un savoir intégrer, mobiliser et transférer un ensemble de ressources (connaissances, savoirs, aptitudes, raisonnements, etc.) dans un contexte donné pour faire face aux différents problèmes rencontrés ou pour réaliser une tâche ". II ajoute et en cela se distingue fondamentalement de Weinert, « il n'y a de compétence que de compétence en acte [...] La compétence ne réside pas dans les ressources (connaissances, capacités, etc.) à mobiliser mais dans la mobilisation même de ces ressources. ”

On peut observer deux types de définition, celles qui mettent en avant le contexte et la situation (compétence en acte) et celles où la compétence préexiste dans la tête du sujet. Une autre catégorisation réside dans le type de situations (déjà rencontrées ou nouvelles): B. Rey utilise ce critère dans l'idée d'une gradation de ces compétences.

Par rapport aux standards proprement dits, on peut en recenser essentiellement trois types dans la littérature internationale, selon leur point de centration (input ou processus de l'apprentissage scolaire contre output) :

- les standards de contenus qui font plutôt référence aux résultats scolaires en se centrant sur les objectifs et les contenus d'apprentissages ; 
- les standards de performance, basés eux aussi sur les résultats mais qui se situent plutôt au niveau des valeurs du test et sont marqués sur une échelle de score ;

- les standards d'opportunity-to-learn qui se rapportent aux input et processus de l'apprentissage scolaire et décrivent les programmes, le personnel et autres ressources qui doivent être mis à la disposition des établissements et des régions scolaires.

On peut aussi choisir des niveaux-cibles variés: un niveau basique à atteindre par une grande majorité d'élèves (standards minimum), un niveau idéal ou encore un niveau moyen d'exigences (standards réguliers).

Le choix qui a été fait en Suisse est de définir des standards de formation minimaux. Ces «standards de formation formulent des exigences à l'égard de l'enseignement et de l'apprentissage scolaire. Ils désignent les objectifs du travail pédagogique, en les exprimant en termes de résultats scolaires attendus. Ils concrétisent ainsi la mission éducative que doivent remplir les écoles de formation générale " (Klieme et al., 2003, p. 16). Pour Klieme, ces standards doivent présenter certaines caractéristiques :

- ils doivent donc se référer aux objectifs de l'évaluation et les transposer en exigences concrètes car les objectifs de l'éducation sont le plus souvent des définitions assez générales des contenus de savoirs, des capacités et des aptitudes visés ;

- les objectifs sont concrétisés sous forme de compétences exigées au travers de modèles de compétences qui exposent les aspects, les degrés et les progressions des compétences. Ces compétences se différencient de ce qu'on trouve dans les plans d'études (listes de matières et de contenus d'enseignement) et nécessitent que l'on identifie des dimensions fondamentales des processus d'apprentissage dans un domaine d'enseignement ou une discipline ;

- enfin, en tant que résultats des processus d'apprentissage, ils doivent être concrétisés sous forme d'exercices et de situations-problèmes ainsi que de procédés fiables permettant de saisir le niveau de compétence réellement atteint par les élèves.

En d'autres termes, ils décrivent des compétences fondées sur un modèle et des niveaux de compétences (progression) validés de manière empirique et doivent être suffisamment concrets pour être vérifiés et mesurés à partir de tests validés.

\section{Limites et problèmes}

Comme on a pu le constater, seuls quatre domaines ont été retenus, ce qui a un côté relativement réducteur. Par ailleurs, ces standards de formation doivent être mesurables pour tous les élèves ce qui constitue aussi un obstacle important (par exemple évaluer les compétences à l'oral et notamment dans des situations d'interaction). Enfin, on parle de standards minimaux ce qui peut, comme l'ont relevé K. Muller et R. Silver (2005) dans leur étude critique sur les standards, conduire à plus long terme à une baisse du niveau d'exigences.

Une autre difficulté réside dans l'aspect national de l'opération. Les standards de formation sont censés se baser sur les objectifs scolaires définis dans les curricula. Or la Suisse possède vingt-six systèmes scolaires différents et trois régions linguistiques. Les conceptions théoriques sous-jacentes peuvent être pas mal contrastées: les unes s'inspirant de courants francophones (France, Québec ou Belgique), les autres des courants germanophones (Allemagne principalement) et les derniers davantage de courants italiens. Deux exemples peuvent être donnés à titre d'illustration : la Suisse romande a déjà fait un travail de coordination et a élaboré un Plan d'étude-cadre romand (PECARO) sur la base de plans d'études de sept cantons défini en terme de compétences ; dans la partie germanophone, ce travail reste à faire (et le sera sur la base des standards). Dans les domaines disciplinaires, il y a des différences importantes d'approches: par exemple, en mathématiques la Suisse romande donne une place importante à la résolution de problèmes ce qui n'est pas le cas dans la partie germanophone. Par ailleurs, dans d'autres cas, certaines notions telle que la proportionnalité (notamment les fractions) sont abordées à l'école primaire dans la partie germanophone ce qui n'est pas le cas en Suisse romande, on observe l'inverse dans le cas de la géométrie et plus particulièrement de l'approche de l'espace. Dans un même ordre d'idées, en Suisse romande, un modèle didactique autour des genres textuels (Dolz \& Schneuwly, 1996) a un impact important sur la conception de l'enseignement de la langue, alors que cette dimension n'est pas présente dans les autres régions. Ceci constitue un paradoxe puisqu'on doit à la fois s'inspirer des programmes scolaires tout en étant confrontés, selon les régions, à la prépondérance de courants et modèles théoriques différents, voire contradictoires. Soulignons que les concepteurs de l'enquête PISA ont pris le parti de se détacher des aspects programmatiques contrairement à d'autres enquêtes internationales menée par I'IEA (Reading literacy en 1991 ou TIMSS en 1995). 


\section{Un exemple : la langue I (langue d'enseignement)}

Un modèle de compétences a été défini dans le domaine de la langue I valable pour les trois langues d'enseignement (allemand, français et italien). Pour ce faire, à l'intérieur de ce domaine, quatre sousdomaines (skills) ont été définis selon une terminologie courante sur le plan international : lire et écouter (réception) ainsi qu'écrire et parler (production).

Le modèle prévoit trois niveaux de description: 1) les aspects de l'activité langagière ; 2) la description des compétences dans les quatre sousdomaines ; 3) la description des tâches.

Pour P. Sieber, responsable du domaine Langue 1 dans le projet HarmoS (2005), les actions langagières sont le point de départ de la description des compétences. Elles sont réalisées dans différentes modalités (production ou réception ) et font appel à différents médias (oral et écrit, communication directe et indirecte). II définit l'activité langagière en cinq aspects qui ne sont pas hiérarchiques et qui interviennent de façon variable selon les situations : situer (reconnaître ou appliquer la fonction de l'activité langagière), planifier (pondérer le poids relatif des différents éléments de contenu, les structurer, les inscrire dans le genre textuel choisi, etc.), réaliser (il s'agit de l'activité de réception ou de production à proprement parler), évaluer (réfléchir et évaluer) et réparer (réviser un texte ou relire le texte pour vérifier et compléter sa compréhension). Ces aspects recouvrent partiellement les sous-échelles utilisées dans PISA (retrouver une information, développer une interprétation et réfléchir sur le texte). II faut toutefois préciser que le modèle défini ici doit prendre en compte à la fois la réception et la production. Or, ces compétences partielles, tout à fait adéquates dans le cas de la littéracie (compréhension de l'écrit ou lecture) dans le sens défini pour l'enquête PISA, à savoir la capacité à comprendre et utiliser des textes écrits ainsi qu'à réfléchir à leur propos, n'auraient pas été adaptées pour les quatre sous-domaines et en particulier, écrire et parler. C'est pourquoi dans le modèle de compétences élaborés pour la langue I dans HarmoS on a choisi de considérer des aspects qui dépassent le cadre de la littéracie (réception) et englobent à la fois la réception et l'expression.

Des compétences partielles, dont on trouvera la description complète en annexe, ont été, entre autres, définies pour la lecture, en fonction des cinq aspects retenus. Ainsi, concernant Situer, on trouve entre autres, des compétences telles que s'adapter aux situations de communication ou reconnaître la fonction du texte. Concernant Planifier, il s'agit notamment de définir sa propre situation par rapport à la lecture et de s'organiser (identifier l'organisation du texte - macrostructure, éléments typographiques). Réaliser comporte de nombreuses compétences partielles, parmi lesquelles : réaliser les correspondances phonèmes-graphèmes ; mobiliser ses compétences langagières (syntaxiques et sémantiques), verbales et non-verbales (organisateurs, anaphores, temps verbaux, etc.) ; interpréter, réaliser des inférences et établir des relations causales; ou encore retrouver des informations explicites et implicites. L'aspect Réparer est plus difficile à prendre en compte dans le cas de la lecture : il peut être considéré comme la compétence à contrôler sa lecture (construction du sens) et à revenir sur celle-ci. Enfin, Évaluer peut être considéré comme comportant les compétences à dégager les éléments les plus importants, à résumer ou à réfléchir par rapport au contenu et à la forme du texte, à analyser la lecture (plans émotionnel, cognitif et métacognitif), et à identifier l'intention de l'auteur.

On notera que certaines compétences partielles sont les mêmes que dans PISA. Ainsi, retrouver une information et développer une interprétation sont inclues dans l'aspect réaliser, et réfléchir sur le texte est une composante d'évaluer.

\section{EN GUISE DE CONCLUSION}

Les enquêtes internationales du type PISA contribuent à une meilleure connaissance de nos systèmes éducatifs à travers la comparaison. Elles peuvent même permettre des exploitations plus locales. Cependant, ces deux types d'exploitation nécessitent une contextualisation qui prend en compte certaines limites de ces enquêtes, notamment lors du passage du niveau international au niveau local ou réciproquement. II faut également garder à l'esprit que ces instruments, aussi sophistiqués qu'ils soient, ne peuvent pas prendre en compte tous les aspects de l'éducation. Ils ne sont pas non plus des instruments universels à même de répondre à toutes les questions que l'on pourrait se poser dans le domaine de l'éducation. Les exemples présentés dans cet article montrent quelques-unes de ces limites.

Cependant, la tentation est forte d'utiliser le même type de démarche dans un contexte national ayant d'autres objectifs. C'est ce qui est arrivé en Suisse. En effet, les résultats de l'enquête PISA 2000 ont donné une impulsion décisive à un projet relativement 
ambitieux d'harmonisation de la scolarité et notamment au volet visant la définition de standards minimaux nationaux. Ces standards nécessitent le développement d'instruments qui s'inspire largement d'une approche de type PISA. Or, PISA est une évaluation qui permet de comparer à un moment donné de la scolarité les compétences des élèves dans trois domaines spécifiques sur la base d'un référentiel de compétences attendues " universelles » communes, définies sans lien direct avec les programmes d'enseignement.

Dans le cas d'un projet du type «HarmoS », les objectifs sont différents, il ne s'agit plus de comparer des entités nationales ou locales mais de pilotage et de régulation du système éducatif ou plus exactement de plusieurs systèmes éducatifs ayant des langues et des cultures différentes par la définition de standards mesurables. II est dès lors nécessaire de définir des compétences qui tiennent compte des programmes scolaires tout en prenant en compte leur dimension variée, tâche qui s'avère complexe et délicate. Par ailleurs, une autre difficulté réside dans l'aspect mesurable, lequel est forcément réducteur par rapport aux contenus curriculaires enseignés à l'école.

De plus, au moment où il s'agit d'élaborer des tâches, on est confronté à un autre type de difficulté : certains types de question sont plus faciles à utiliser (questions à choix multiples ou questions à réponse fermée contra questions ouvertes où le correcteur devra interpréter la réponse de l'élève). Or, les résultats de PISA aussi bien en littéracie qu'en mathématiques ont montré l'effet du type de questionnement. De plus, le type de test retenu sera de préférence écrit, ce qui a forcément des incidences sur les domaines mesurés : par exemple la production orale (et en particulier en interaction) ou l'expérimentation en sciences.

Pour conclure, PISA a permis des apports non seulement en termes de connaissance des systèmes éducatifs mais également du point de vue des méthodes et de leur implémentation. Cependant, il faut rester conscient des limites de ce type d'enquêtes. En effet, l'élaboration de standards nécessite le développement d'instruments plus diversifiés pour cerner les objectifs variés de formation visés par les systèmes scolaires qui dépassent largement les compétences prises en compte dans les enquêtes comparatives internationales.

Jean Moreau

jean.moreau@unil.ch

Unité de recherche pour le pilotage des systèmes pédagogiques (URSP), Lausanne

Christian Nidegger christian.nidegger@etat.ge.ch Service de la recherche en éducation (SRED), Genève

Anne Soussi

anne.soussi@etat.ge.ch Service de la recherche en éducation (SRED), Genève

\section{NOTES}

(1) Un élève de compétence $x$ a $50 \%$ de chance (dans PISA 2000 $62 \%)$ de répondre correctement à l'item de difficulté $x$.

(2) Par exemple en 2003, l'ensemble des items a été réparti dans treize cahiers. Chaque item apparaît dans quatre cahiers différents. Les élèves ne répondent qu'à un des treize cahiers.
(3) Cette instance permanente regroupe les chefs de départements (ministres) chargés de l'instruction publique des vingt-six cantons en vue de coordonner leur action sur la base d'accords entre les cantons.

\section{BIBLIOGRAPHIE}

AlLAL L. (1999). "Acquisition et évaluation des compétences en situation scolaire ". Raisons éducatives, $\mathrm{n}^{\circ} 2$ ("L'énigme de la compétence »), p. 77-94.

Brol A.-M. ; Moreau J. ; Soussi A. \& Wirthner M. (2003). Les compétences en littératie. Rapport thématique de l'enquête PISA 2000. Neuchâtel : Office fédéral de la statisitique (OFS) ; Conférence suisse des directeurs cantonaux de l'instruction publique (CDIP).

Confédération HelvétiQue : ConfÉrence suisse des diRECTEURS CANTONAUX DE L'INSTRUCTION PUBLIQUE (2004). HarmoS: finalités et conceptions du projet (juin 2004). Berne: CDIP. Document au format PDF disponible sur Internet à l'adresse : <http://www.edk.ch/PDF Downloads/Harmos/Harmos_Weissbuch_f.pdf> (consulté le 23 janvier 2007).

CrAHAY M. (2006). "Dangers, incertitudes et incomplétude de la logique de la compétence en éducation ". Revue française de pédagogie, $\mathrm{n}^{\circ} 154$, p. 97-110.

Dolz J. \& SCHNEUWly B. (1996) «Genre et progression en expression écrite : éléments de réflexion à propos d'une expérience romande ", Enjeux, n³7/38, p. 49-75.

EMbretson S. E. \& ReISE S. P. (2000). Item response theory for psychologists. Mahwah : L. Erlbaum. 
KLIEME E. et al. (2003). Expertise. Zur Entwicklung nationaler Bildungsstandards Bonn: Bundesministerium für Bildungs und Forschung [BMBF] : Referat Publikationen. Document au format PDF disponible sur Internet à l'adresse : <http://www.bmbf.de/pub/zur_entwicklung nationaler_bildungsstandards.pdf> (consulté le 23 janvier 2007).

Le Boterf G. (1995). De la compétence : essai sur attracteur étrange. Paris : Éd. d'Organisation.

JoNNAERT P. (2002). Compétences et socioconstructivisme: un cadre théorique. Bruxelles : De Boeck.

MüLLER K. \& SiLVER R. (2005). "Standards in education. Review of US literature ". In Matthis Behrens (coord.), Analyse de la littérature critique sur le développement, l'usage et l'implémentation de standards dans un système éducatif. Neuchâtel : Institut de recherche et de documentation pédagogique.

NIDEgGeR C. [coord.] (2005). PISA 2003 : Compétences des jeunes romands : Résultats de la seconde enquête PISA auprès des élèves de $9^{e}$ année. Neuchâtel : Institut de recherche et de documentation pédagogique.

OCDE (2003). Cadre d'évaluation de PISA 2003. Connaissances et compétences en mathématiques, lecture, science et résolution de problèmes. Paris : OCDE.

OECD (2004). Learning for tomorrow's world: First results from PISA 2003. Paris : OECD.

OECD (2005). Pisa 2003 technical report. Paris : OECD.

Rey B.; Carette V.; Defrance A. \& Kahn S. (2003). Les compétences à l'école. Bruxelles : De Boeck.

RoEgIERs X. (2000). Une pédagogie de l'intégration : compétences et intégration des acquis dans l'enseignement. Bruxelles : De Boeck.

Sieber P. et al. (2005). Schritte zu einem Kompetenzmodell Erstprache. (document provisoire).

WEINERT F. E. (2001). "Vergleichende Lestungsmessug in Schulen: eine umtrittene Selbstverständlichkeit ». In F. E. Weinert (éd.), Leistungsmessungen in Schulen. Weinheim \& Basel : Beltz Verlage, p. 17-31.

\section{Annexe. - Compétences partielles dans le domaine de la lecture}

\begin{tabular}{|c|c|}
\hline \multirow{4}{*}{ situer } & - repérer le thème principal et le contenu central \\
\hline & - reconnaître la fonction du texte (en lien avec les genres textuels) \\
\hline & - s'adapter aux situations de communication \\
\hline & - trouver l'intérêt de la lecture en liaison avec le texte \\
\hline \multirow{3}{*}{ planifier } & $\begin{array}{l}\text { - définir sa propre situation par rapport à la lecture et s'organiser (identifier l'organisation du texte } \\
\text { (macrostructure, éléments typographiques) }\end{array}$ \\
\hline & - établir les particularités du texte \\
\hline & - construire un projet de lecture et choisir les stratégies de lecture \\
\hline \multirow{9}{*}{ réaliser } & - réaliser les correspondances phonèmes-graphèmes \\
\hline & - identifier et décoder au niveau des mots et des phrases \\
\hline & $\begin{array}{l}\text { - mobiliser ses connaissances langagières (syntaxiques et sémantiques), verbales et non-verbales } \\
\text { (organisateurs, anaphores, temps verbaux, etc.) }\end{array}$ \\
\hline & - décoder les représentations iconographiques \\
\hline & - mobiliser les pré-requis et expériences, connaissances du monde \\
\hline & $\begin{array}{l}\text { - interpréter : réaliser des inférences et établir des relations causales, établir des liens entre } \\
\text { différentes parties du texte, construire une représentation mentale, créer l'imaginaire, établir une } \\
\text { cohérence }\end{array}$ \\
\hline & - retrouver des informations implicites et explicites \\
\hline & - développer une proximité émotionnelle : concernant le thème, les personnages et d'autres éléments \\
\hline & $\begin{array}{l}\text { - identifier les éléments esthétiques, les figures de style et les structures et les utiliser pour } \\
\text { construire le sens }\end{array}$ \\
\hline réparer & - contrôler sa lecture (construction du sens) et revenir sur sa lecture \\
\hline \multirow{5}{*}{ évaluer } & - dégager les éléments les plus importants, résumer \\
\hline & - récapituler l'expérience en lecture et évoquer sa compréhension du texte \\
\hline & $\begin{array}{l}\text { - réfléchir par rapport au contenu et à la forme, analyser la lecture (plan émotionnel, cognitif et } \\
\text { méta-cognitif), identifier l'intention de l'auteur }\end{array}$ \\
\hline & - échanger avec d'autres à propos de l'expérience de la lecture et la signification du texte \\
\hline & - justifier son point de vue, argumenter \\
\hline
\end{tabular}

